\title{
The Critical Role of Hippocampal Dopamine in the Pathogenesis of Hepatic Encephalopathy
}

\author{
Baiyi CHEN ${ }^{1,2}$, Yang YANG ${ }^{1}$, Shijia LI $^{1}$, Xiuqi ZHU ${ }^{1}$, Ying QI ${ }^{1}$, Feng HONG \\ ${ }^{1}$ School of Preclinical Medicine, Wannan Medical College, Wuhu, China, ${ }^{2}$ Keck School of \\ Medicine, University of Southern California, Los Angeles, CA, USA
}

Received August 25, 2020

Accepted December 3, 2020

Epub Ahead of Print January 14, 2021

\section{Summary}

The pathogenesis of hepatic encephalopathy (HE) has been generally linked to blood ammonia, gamma-aminobutyric acid and serotonin. However, the exact mechanism remains unclear. In the present study, we aimed to explore the role of hippocampal dopamine (DA) and its receptors in the pathogenesis of $\mathrm{HE}$ through the use of behavioral testing, western blotting, and immunofluorescence staining in normal rats, HE model rats and rats treated with the DA precursorlevodopa (L-DOPA). HE model rats manifested fibrotic livers and showed serious behavioral disorders. They also had significantly lower hippocampal DA content and increased expression of both D1 and D2 receptors relative to normal rats. After treatment with L-DOPA, the HE model rats showed normal behavior and expression of D1 returned to normal levels. Furthermore, pretreatment with the $\mathrm{D} 1$ antagonist $\mathrm{SCH} 23390$ blocked the therapeutic effect of L-DOPA on behavior in HE model rats. Taken together, these results clarify that the decrease in hippocampal DA plays a role in the pathogenesis of $\mathrm{HE}$ and that this effect is mediated by $D 1$. These findings provide new evidence for the pathogenesis of HE.

\section{Key words}

Hepatic encephalopathy $\bullet$ Hippocampal DA $\bullet$ D1 receptor $\bullet$ L-DOPA

\section{Corresponding author}

F. Hong, School of Preclinical Medicine, Wannan Medical College, No. 22 Wenchang West Road, Wuhu 241002, China. E-mail: hongfengshengli@163.com

\section{Introduction}

Hepatic encephalopathy (HE) is a form of central nervous system dysfunction caused by severe liver disease (Dharel et al. 2015). The main clinical symptoms of HE include disturbance of consciousness, behavioral disorder and coma (Kornerup et al. 2018). The survival rate for $\mathrm{HE}$ is only $23 \%$ after three years (Ellul et al. 2015). HE significantly affects normal brain function, results in the development of hypertrophic astrocytes and alters gray matter structural network (Zou et al. 2018). The pathogenic mechanisms of $\mathrm{HE}$ remain unclear. Several possible mechanisms include ammonia poisoning (Rössle et al. 1984), pseudo neurotransmitters (Davis and Bajaj 2018), and $\gamma$-aminobutyric acid (Leke et al. 2011). Of these, ammonia poisoning is the dominant hypothesis, which posits that increased ammonia concentrations in the blood of HE patients results in neuronal dysfunction (Ochoa-Sanchez and Rose 2018). However, approximately $31 \%$ of HE patients show no increases in blood ammonia levels (Ninan and Feldman 2017), which indicates that the ammonia poisoning hypothesis cannot fully explain the pathogenesis of HE.

The hippocampus, which can be functionally divided into dorsal, intermediate, and ventral parts (Yang and Wang 2017, Riljak et al. 2020), is a brain structure that regulates spatial cognition, balance control, emotion (de Voogd et al. 2019), addiction (Canales 2013), and memory and learning (Souza-Braga et al. 2018). Hippocampal abnormalities can lead to behavioral disorders such as spatial cognitive anomalies and balance disorders (Anand and Dhikav 2012). Damage to 
hippocampus has been reported in HE patients who show impaired learning, memory, and connectivity functions (García-García et al. 2018).

Dopamine (DA) is a catecholamine neurotransmitter that is widely distributed in the substantia nigra, the ventral tegmental area of the midbrain and the hypothalamus (Yang and Wang 2017). DA regulates feeling and excitement, motor function, addiction, learning and memory (Tang and Dani 2009, Klein et al. 2019) via its receptors, which are termed $\mathrm{D} 1$ and $\mathrm{D} 2$. DA dysfunction in the extracorporeal vertebral column is commonly observed in patients with hepatic coma (Shawcross 2018, Delis et al. 2013), which is a severe clinical symptom of HE (Kornerup et al. 2018). Interestingly, administration of the precursor of DA, L-DOPA, through a nasogastric tube can awaken patients with hepatic coma (Tyce et al. 1983). Hippocampal DA, which mainly derives from the ventral tegmental area and substantia nigra (Cavus et al. 2005), is associated with addiction and cognitive development (El-Ghundi et al. 2007). In addition, destruction of hippocampal neurons has been reported in mice with HE induced by alcohol combined with carbon tetrachloride, which is mainly regulated by increased TNF- $\alpha$ and IL-10 (Azhari and Swain 2018). It has also been demonstrated that IL-10 can inhibit DA secretion (Li et al. 2018), which further suggests that liver fibrosis may induce decreases in DA. Taken together, these findings suggest that hippocampal DA may decrease in liver fibrosis and result in the development of HE.

To further explore this possibility, in the present study, HE models were established to explore changes in hippocampal DA and D1/D2 receptors in the rodent brain. We then explored the treatment effect of DA on the behavior of HE model rats after injection with L-DOPA. Lastly, rats were pretreated with the D1 antagonist SCH23390 to explore the mechanism through which DA protects against behavioral disorders.

\section{Methods}

\section{HE model rats}

All experiments were approved by the Animal Experimental Ethics Committee of Wannan Medical College following the Administration Regulations on Use of Laboratory Animals in Anhui Municipality. All animals were raised in a standard animal care facility on a $12 \mathrm{~h}$ light/dark cycle with access to food and water. The light was turned on at 6:00 am and off at 6:00 pm. Male
Sprague-Dawley rats weighing 180-200 g (8-week-old) were randomly divided into a normal group $(n=10)$, model group $(n=20)$, L-DOPA treatment model group (L-DOPA group) $(\mathrm{n}=15)$, and $\mathrm{SCH} 23390$ pretreatment L-DOPA treatment model group (SCH23390 group) $(n=5)$ (Table 1). Rats in the model group, L-DOPA group, and $\mathrm{SCH} 23390$ group were given a mixed solution containing $40 \% \mathrm{CCl}_{4}$ (Bengbu Chemical Reagent Factory, China) and olive oil (Olivoila, China) $\left(\mathrm{CCl}_{4}\right.$ :olive oil=1:1.5) by abdominal subcutaneous injection. Each rat was administered a mixed solution $5 \mathrm{ml} \cdot \mathrm{kg}^{-1}$ on the first injection, followed by a mixed solution $3 \mathrm{ml} \cdot \mathrm{kg}^{-1}$ twice a week for a total of 8 weeks (Wang et al. 2017). The normal group was intraperitoneally injected with saline twice per week for 8 weeks. Rats in the L-DOPA group were administered with L-DOPA (AmyJet Scientific, China) at $10 \mathrm{mg} \mathrm{kg}^{-1}$ starting at week 9 and receiving intraperitoneal injections for 4 weeks. Rats in the $\mathrm{SCH} 23390$ group were intraperitoneally injected with $\mathrm{SCH} 23390$ (Beyotime Biotechnology, China)-a D1 antagonist at $0.5 \mathrm{mg} \cdot \mathrm{kg}^{-1}$, 30 min before treatment with L-DOPA (Beauvais et al. 2010). Rats' weights were monitored throughout the experiment. The behavioral tests were conducted at 8 weeks and 12 weeks since the first drug injection at 3:00 pm. After the rats were sacrificed at 12 weeks, the liver, fat, and hippocampus were immediately removed for analysis.

\section{Animal behavior scoring test}

We used the Animal Behavior Scoring Test table (Wang et al. 2017) to evaluate the behavior and mortality of the rats in each group. Behaviors were scored on sixpoint scale as follows: +++ means rat shows normal behavior; ++ means rat shows mild lethargy; + means rat decreased motor activity, poor gesture control, and diminished pain perception; - means rat has severe ataxia and no spontaneous righting reflex; -- means rats has no righting reflex and no reaction to pain stimuli; and --- means rat has death or zero motor activity. The scores and behaviors that the rats showed during the test were recorded.

\section{Beam balance test}

In line with previous reports (Li et al. 2012), a rectangular beam measuring $80 \mathrm{~cm}$ in length was used in the beam balance test. The beam was laid flat about $10 \mathrm{~cm}$ above the ground, with a safe cage at the end of the beam. After the rats adapted to walking on a $5 \mathrm{~cm}$ width beam, 
a narrow beam of $2.5 \mathrm{~cm}$ width was used for the test. The rats were allowed to walk on the beam, and the time and performance were recorded in detail. Performances were marked on a six-point scale with a higher score representing greater dysfunction: $0=$ maintained balance on the beam, walked on the top without falling; $1=$ grasped the side of the balance beam; $2=$ grasped the side of the balance beam, with one limb falling off the beam; $3=$ grasped the beam with two limbs falling off the beam or hanging from the beam ( $>60 \mathrm{~s}) ; 4=$ attempted to balance on the beam but fell off $(>40 \mathrm{~s}) .5=$ attempted to balance on the beam but fell off $(>20 \mathrm{~s})$; and $6=$ no attempt to maintain balance or hang from the beam $(>20 \mathrm{~s})$. The normal score is 0 , whereas maximal disruption of behavior is scored 6 . We recorded the time, score, and circumstances for every rat that completed the test.

Table 1. Rat groups and drug injection.

\begin{tabular}{|c|c|c|c|c|}
\hline & Normal group & Model group & $\begin{array}{c}\text { Model+L-DOPA } \\
\text { group }\end{array}$ & $\begin{array}{c}\text { Model+SCH23390+ } \\
\text { L-DOPA group }\end{array}$ \\
\hline Saline & + & & & \\
\hline $\mathrm{CCl}_{4}$ :olive oil $(1: 1.5)$ & & + & + & + \\
\hline$L-D O P A$ & & & + & + \\
\hline SCH23390 & & & & + \\
\hline
\end{tabular}

\section{Hematoxylin-eosin (H\&E) staining}

Liver tissue extracted from the rats was dehydrated using $15 \%$ sucrose solution and $30 \%$ sucrose solution and then fixed with $4 \%$ paraformaldehyde. The tissue was embedded in the optimal cutting temperature compound (Sakura, China) and cut into $6 \mu \mathrm{m}$ sections at $-20{ }^{\circ} \mathrm{C}$. The sections were washed in the phosphate buffer containing $0.3 \%$ Triton X-100 (PBST) (Zsbio Commerce Store, China) solution. The sections were immersed in the hematoxylin solution for $1 \mathrm{~min}$ and $40 \mathrm{~s}$, rinsed with tap water for $2 \mathrm{~min}$, and differentiated with $1 \%$ hydrochloric acid alcohol solution for 2-3 s until the sections turned blue. The sections were then observed under a microscope.

\section{Masson's trichrome staining}

Liver tissue was embedded in the cutting temperature compound (Sakura, China) and cut into $6 \mu \mathrm{m}$ sections at $-20{ }^{\circ} \mathrm{C}$. The sections were treated using Masson's Trichrome Stain Kit (Leagene, China) and incubated with iron hematoxylin solution for $7 \mathrm{~min}$, acidic ethanol for $40 \mathrm{~s}$, magenta and Ponceau mixed solution for $11 \mathrm{~min}$, phosphomolybdic acid for $50 \mathrm{~s}$, and aniline blue for $1 \mathrm{~min}$. The sections were then observed under a microscope.

\section{Western blot (WB)}

Liver or hippocampus tissue was homogenized in the cold lysis buffer comprising a mixture of protease inhibitor cocktail (Beyotime Biotechnology, China). For each sample, $50 \mathrm{mg}$ was extracted and subjected to electrophoresis using $10 \%$ polyacrylamide gel and then transferred to a nitrocellulose membrane. We used $5 \%$ nonfat-milk diluted in Tris-buffered saline solution to block the nonspecific binding sites for $1 \mathrm{~h}$. The membranes were then incubated with primary antibodies (AmyJet Scientific, China) against D1, D2, and caspase-3 (CASP-3) overnight at $4{ }^{\circ} \mathrm{C}$. After washing with Trisbuffered saline Tween 20 (TBST), membranes were incubated with secondary antibodies (AmyJet Scientific, China) for $2 \mathrm{~h}$ at room temperature. Membranes were visualized using an Odyssey Infrared Imager.

\section{Immunofluorescence staining}

Brain tissue was embedded in the optimal cutting temperature compound (Sakura, China) and cut into $20 \mu \mathrm{m}$ sections at $-20^{\circ} \mathrm{C}$. Sections containing the hippocampus region were incubated with $5 \%$ horse serum for $30 \mathrm{~min}$ and then with anti-D1 antibody (Alomone Labs, Jerusalem, Israel) overnight at $4{ }^{\circ} \mathrm{C}$. After washing with PBST, sections were incubated with secondary antibody (Cell Signaling Technology, USA) and DAPI (Sigma, USA) for $2 \mathrm{~h}$. Sections were then observed under an immunofluorescence microscope (Olympus, BX53).

\section{Ultra-performance liquid chromatography/mass} spectrometry (UPLC/MS)

DA content in rat hippocampus was measured by UPLC/MS analysis. Tissue $(50 \mathrm{mg}$ ) was homogenized in $500 \mu \mathrm{l}$ ice cold methanol. The homogenates were centrifuged at $12000 \mathrm{rpm}$ for $20 \mathrm{~min}$ at $4{ }^{\circ} \mathrm{C}$. The 
supernatant was further evaporated until dry under nitrogen blow. The sediments were reconstituted in $200 \mathrm{ml}$ deionized water and this solution was immediately analyzed by UPLC/MS.

\section{Data analysis}

The samples used for data analysis were randomly selected from the normal, model, L-DOPA and SCH23390 group. Data were compared between the three or four groups using analysis of variance (ANOVA). Statistical analyses were performed using GraphPad Prism 6.0 software. $\mathrm{p}<0.05$ was considered statistically significant.

\section{Results}

\section{Characterization of liver fibrosis in HE model rats}

No significant changes in average body weight
(Fig. 1a), retroperitoneal fat (Fig. 1c), or food intake (Fig. 1d) were observed in model group. However, the rate of body weight change (Fig. 1b) and the appearance of liver tissue significantly changed. Specifically, the liver tissue of the model group was more rugged and fragmentary (Fig. 2a) compared with the normal group. H\&E staining showed that the HE model rat liver had an incomplete, loose cell structure compared with normal rat liver (Fig. 2b). Masson's trichrome staining showed a larger area of collagen deposition in the model group relative to the normal group, which indicated higher severity of hepatic fibrosis (Fig. 2c). However, the liver weight did not significantly change (Fig. 2d). Moreover, WB results demonstrated that the apoptotic factor CASP-3 was significantly increased in the liver of the HE model group (Fig. 2e, $\mathrm{p}=0.0033$ ). Taken together, these results demonstrate that $\mathrm{HE}$ model rats typically developed severe liver fibrosis. a
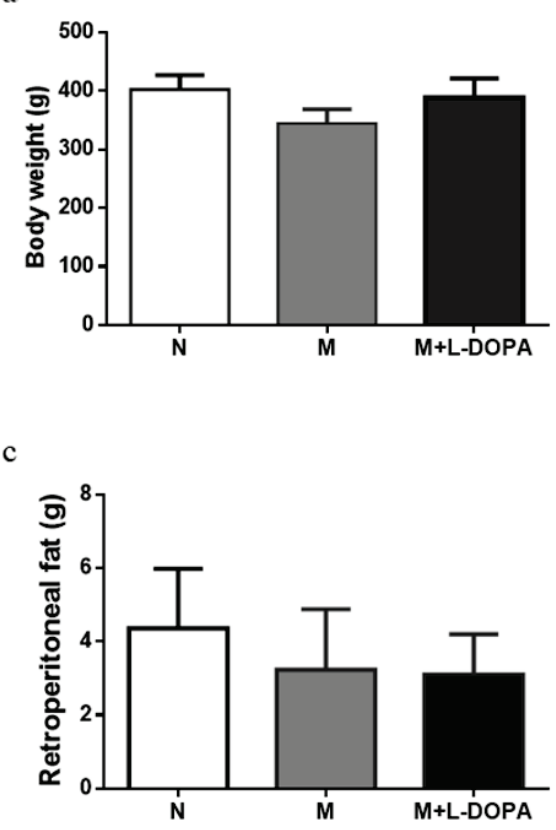

b

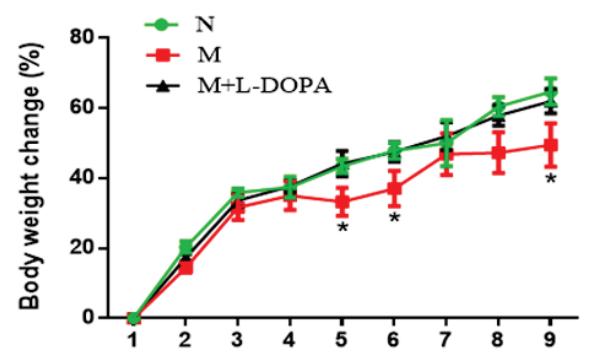

d

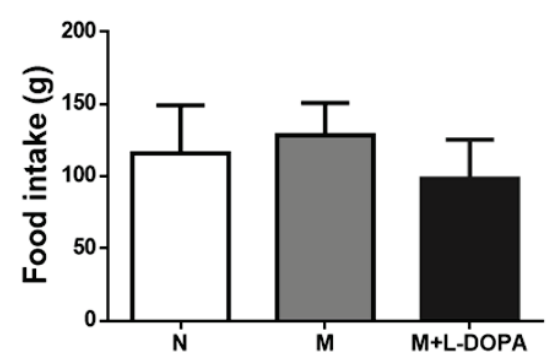

Fig. 1. General physical manifestations of rats. (a) Average body weight of normal, model and L-DOPA treatment rat. (b). Rate of body weight change in modeling and treatment process. The first measure is the beginning of the modeling process, and the fourth measure is the beginning of the treatment process. (c). Average retroperitoneal fat of normal, model and L-DOPA treatment rat. (d). Average food intake of normal, model and L-DOPA treatment rat. N: normal group; M: model group; M+L-DOPA: L-DOPA treatment model group.

\section{Symptoms of HE and changes in hippocampal DA}

Tests showed that the model group lost the ability to maintain balance. The Animal Behavior Scoring Test showed that the model group had a more severe behavior disorder than the normal group (Fig. 3a). The average score on the Beam Balance Test was close to 0 for the normal group and close to 6 for the HE model group (Fig. 3b). The time the model group spent on the balance beam was significantly higher compared with the normal group (Fig. 3c, $\mathrm{p}<0.001$ ). Hippocampal DA content of the model group was significantly reduced (Fig. $3 \mathrm{~d}, \mathrm{p}<0.001$ ) whereas hippocampal expression of D1 and D2 were increased in the model group (Fig. 4a, b, c, d, p<0.05), which indicates that DA reduction in the hippocampus is associated with HE. 


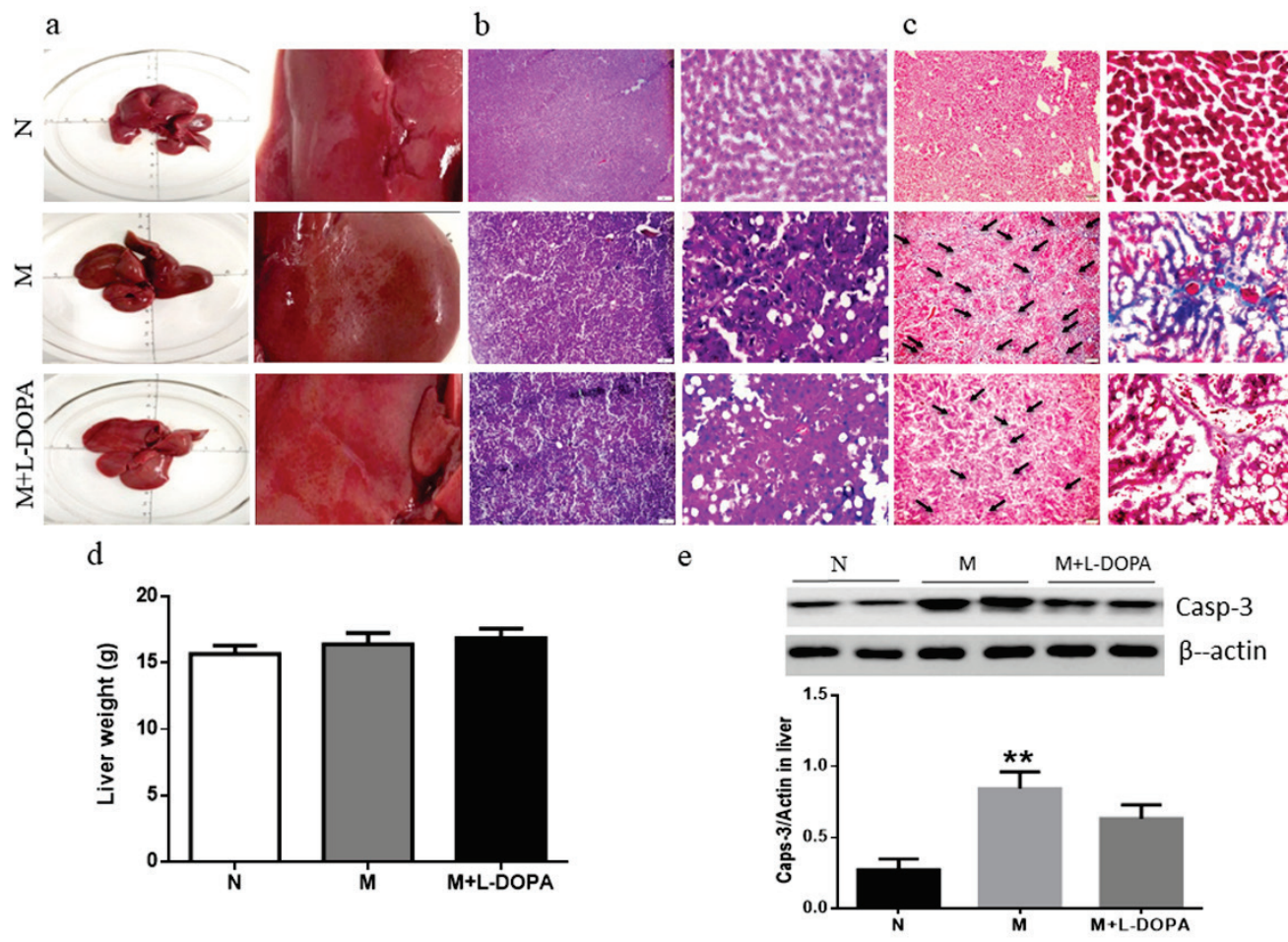

Fig. 2. Inflammation indicated by histology and morphology. (a) Morphology of liver tissues. (b) H\&E staining images of liver sections. (c) Masson's Trichrome Staining images of liver sections. (d) Average liver weight. (e) Protein expression of CASP-3 in liver tissue detected by WB. $* * p<0.01$, compared with the normal group.

a

\begin{tabular}{lcccc}
\hline & $\mathrm{N}$ & $\mathrm{M}$ & $\begin{array}{c}\mathrm{M}+ \\
\text { L-DOPA }\end{array}$ & $\begin{array}{c}\mathrm{M+} \\
\text { SCH23390+ } \\
\text { L-DOPA }\end{array}$ \\
\hline 8 Weeks+1 \\
Day & +++ & --- & --- & -- \\
$\begin{array}{c}8 \text { Weeks+3 } \\
\text { Day }\end{array}$ & +++ & --- & --- & --- \\
$\begin{array}{c}12 \text { Weeks+1 } \\
\text { Day }\end{array}$ & ++ & -- & + & --- \\
$\begin{array}{c}12 \text { Weeks+3 } \\
\text { Day }\end{array}$ & ++ & -- & + & --- \\
\hline
\end{tabular}

c

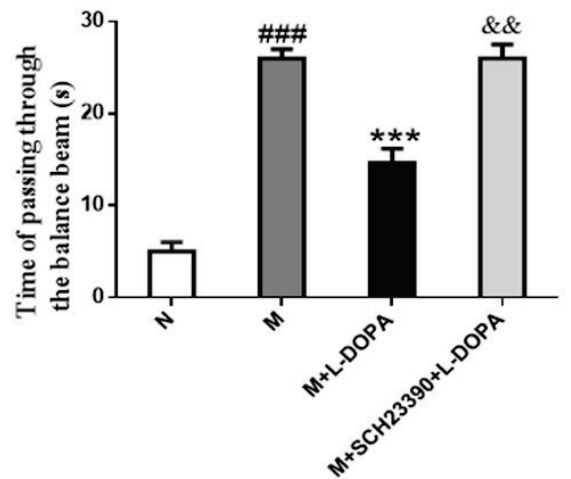

$\mathrm{b}$

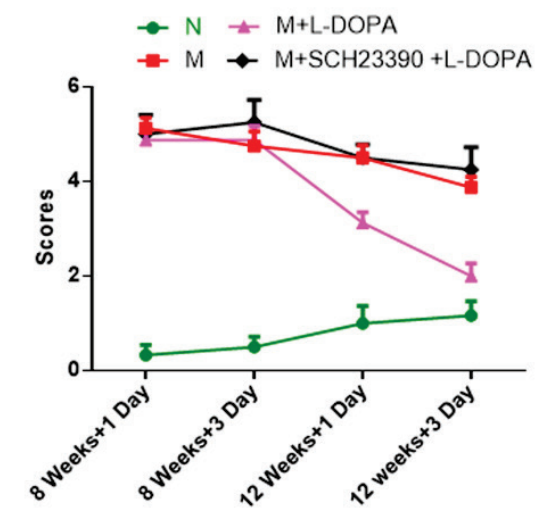

d

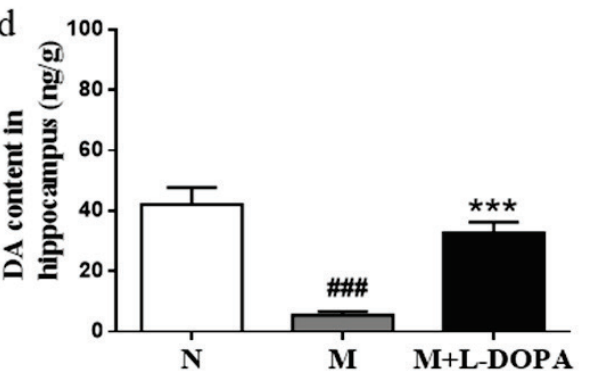

Fig. 3. Behavioral tests and evaluation. (a) Evaluation of Animal Behavior Scoring Test. The symbols " + " and "-" represent the evaluation of each group. (b) Trends in scores change of the beam balance test: evaluation based on the beam balance test scoring criteria. (c) Average time spent on the balance beam. (d) DA content in the hippocampus tissue. The age of rat in a and $b$ is calculated from modeling. M+SCH23390+L-DOPA: SCH23390 group. ${ }^{\# \# \# ~} \mathrm{p}<0.001$, compared with the normal group. $* * * p<0.001$, compared with the model group. ${ }^{\text {\& }} \mathrm{p}<0.01$, compared with the L-DOPA treatment group. 


\section{Therapeutic effect of $L-D O P A$ on $H E$}

To investigate whether DA regulates the pathogenesis of HE, L-DOPA was injected into HE model rats. The L-DOPA group showed improved scores on the Animal Behavior Scoring Test relative to the model group, with performance restored to normal levels (Fig. 3a). The Beam Balance Test scores for the L-DOPA group dramatically decreased to resemble scores for the normal group. Attempts to maintain balance on the beam increased and the ability to keep balance were fortified in the L-DOPA group (Fig. 3b). The time L-DOPA group spent on the balance beam significantly decreased to the normal level (Fig. 3c, $\mathrm{p}<0.001)$. These results indicate that L-DOPA can alleviate HE symptoms in model HE rats.

\section{Mechanism of L-DOPA effects on of behavioral disorders in HE model rats}

To explore the mechanism of DA in the treatment of HE, we evaluated the effects of DA on the liver and hippocampus of model group. The body weight (Fig. 1a), the rate of body weight change (Fig. 1b) and food intake in the L-DOPA group (Fig. 1c) were much closer to those of the normal group. However, L-DOPA treatment did not alleviate the symptoms of liver fibrosis in the model group (Fig. 2a, b, c). Compared with the model group, the L-DOPA group showed no significant changes in liver tissue weight (Fig. 2d). The appearance of liver tissue in the L-DOPA group was relatively flat and complete compared to the model group, however there were still pink spots on the surface, with the color closer to that of the normal group (Fig. 2a). H\&E staining showed that the sections from the L-DOPA group were relatively intact compared to the model group (Fig. 2b). Masson's trichrome staining showed that the L-DOPA group had less collagen deposition in the liver than the model group (Fig. 2c). Furthermore, the CASP-3 content in the L-DOPA group showed no significant decrease compared with the model group (Fig. 2e). These results indicate that the L-DOPA treatment minimally modified liver appearance or function. In hippocampus, L-DOPA treatment significantly increased DA content (Fig. 3d). WB results showed that L-DOPA treatment significantly decreased the high expression of D1 $(p<0.05)$, but not of D2 in HE model rats (Fig. 4a, b, c, d), which indicates the possible role of D1 in L-DOPA treatment for HE. Then we used immunofluorescence staining to image D1 receptors in the hippocampus (Fig. 5). To further demonstrate the role of D1, we pre-treated the HE model rats with the $\mathrm{D} 1$ antagonist $\mathrm{SCH} 23390$. We found that SCH23390 pretreatment blocked the beneficial effects of L-DOPA in the HE model rats (Fig. 3a, b, c, p<0.01). a

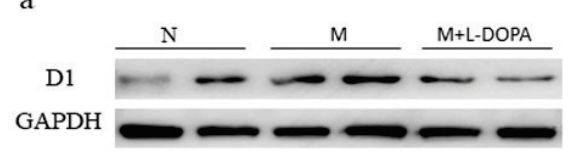

c

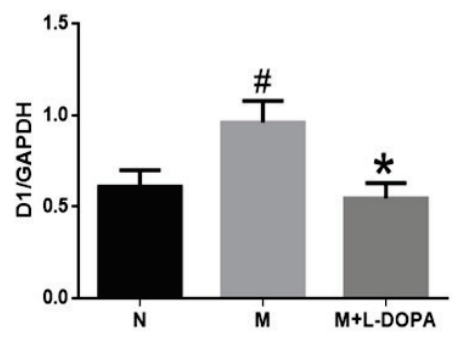

\section{Discussion}

The present study provides the first evidence that DA plays a significant protective role in the pathogenesis of HE. The decrease of hippocampal DA is relevant to the pathogenesis of HE, and L-DOPA treatment significantly improved behavioral disorders in HE model rats. Further,

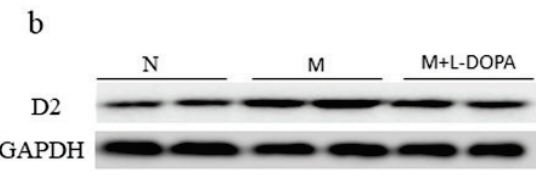

d

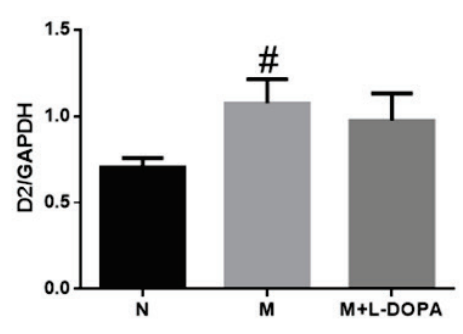

Fig. 4. Expression of $D 1$ and D2 receptors in hippocampus. (a) D1 content in the hippocampus detected by WB. (b) D2 content in the hippocampus detected by WB. (c) Analysis of WB results of D1 content in the hippocampus. (d) Analysis of WB results of D2 content in the hippocampus. \# $\mathrm{p}<0.05$, compared with the normal group. $* p<0.05$, compared with the model group. we showed that this treatment effect on behavioral changes was mediated by the D1 receptor.

In the present study, the appearance of behavioral disorder was observed by modeling liver fibrosis in rats. We first reported that the amount of DA in the hippocampus was significantly decreased. Hippocampal DA plays fundamental roles in learning and 


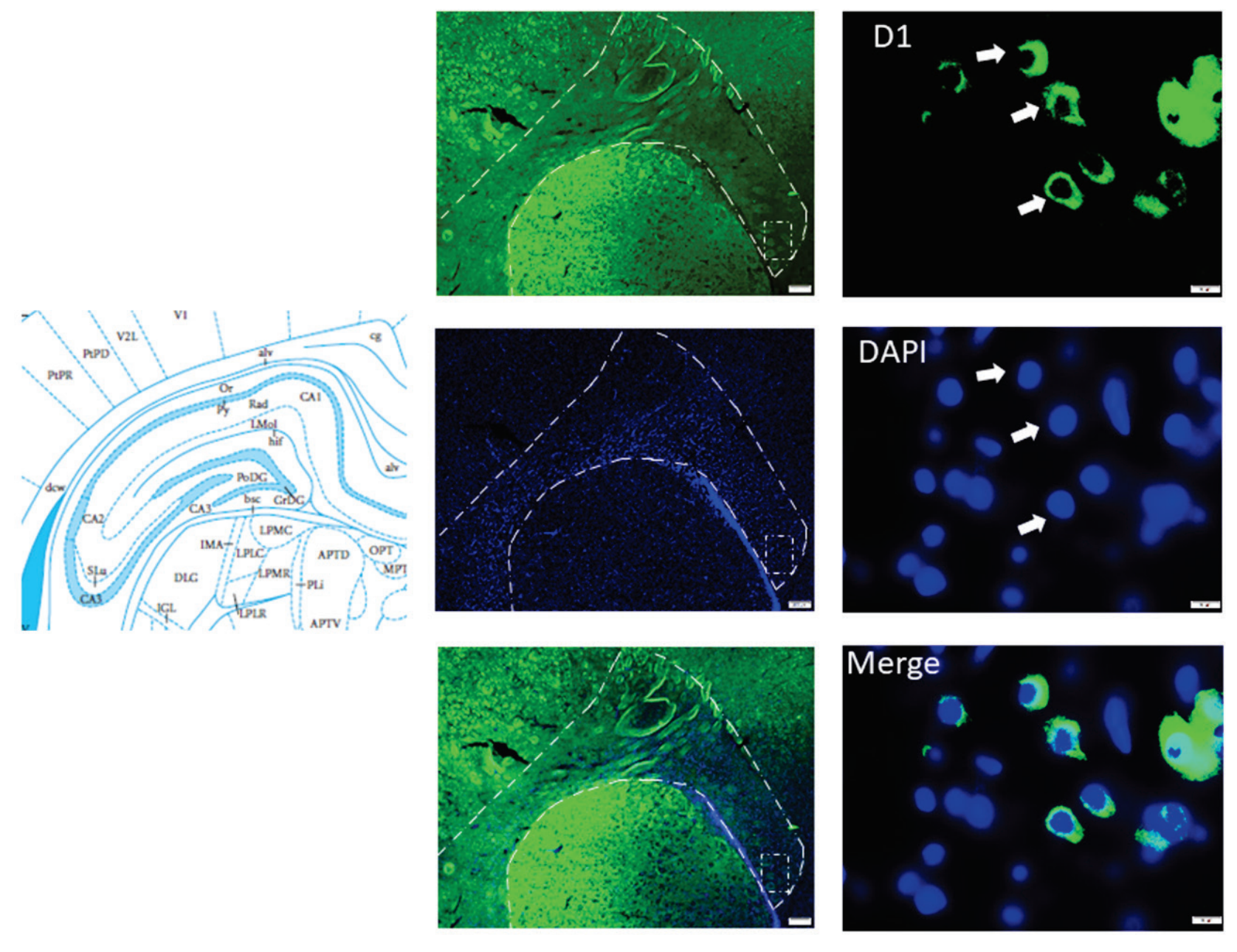

Fig. 5. Immunofluorescence staining of $D 1$ receptors in the hippocampus. Hippocampus is outlined with dotted lines, with the dotted rectangle delineating the area of positive expression of D1 receptors. Green: D1, blue: DAPI (2-(4-Amidinophenyl)-6-indolecarbamidine dihydrochloride).

cognition, regulating emotion and senses (Berke 2018), and addiction (García-García et al. 2018, Tang and Dani 2009). Previous reports have found that significant decreases in hippocampal DA levels can lead to a variety of advanced activity disorders in rats (Knell et al. 1974) and humans (Sil'kis 2012), including hypokinetic movements, muscle rigidity, static tremors, and postural instability, and that these disorders can be improved by DA/L-DOPA (Haddad et al. 2017). Furthermore, reports have suggested that DA dysfunction in the vertebral system is present in patients with hepatic coma (Shawcross 2018, Delis et al. 2013), and that L-DOPA can revive patients with liver coma (El-Ghundi et al. 2007, Knell et al. 1974). In this regard, we conclude that decreased hippocampal DA was related to behavioral disorders in the HE model group.

Nervous system disorders are commonly observed in patients with severe liver disease. In patients with HE, neurological disorders may be induced by toxins from the liver via systemic circulation, which may cross the blood-brain barrier (BBB) and affect the brain (Schwendimann and Minagar 2017). Moreover, in HE mice, increased IL-10 destroys hippocampal neurons, which are mainly regulated by increased TNF- $\alpha$ and IL-10, leading to behavioral disorder (Azhari and Swain 2018). Report has shown that IL-10 inhibits DA secretion (Li et al. 2018), which further suggests that liver fibrosis may induce decreases in DA, and that DA may be involved in the development of HE. Systemic administration of DA in mice has been shown to reduce IL-10 levels in blood, brain, and liver (Matalka et al. 2011). Therefore, we used L-DOPA to elevate DA content in the brain, which may have an effect in alleviating hippocampal injury by reducing the IL-10 level in the brain. Moreover, reports have clarified that systemic administration of L-DOPA can rescue proper functioning of hippocampus nucleus accumbens excitatory synaptic transmission in $\mathrm{Tg} 2576$ mice, thus 
recovering the spatial memory and persistent memory functions of hippocampus (Cordella et al. 2018). Some HE patients show the comparable symptoms with Parkinson's disease, such as slow cerebration, stiffness of movements, and tremors. As a result, it has been speculated that dopamine drugs used as the treatment of Parkinson's disease should be evaluated in HE patients (Haddad et al. 2017).

To further demonstrate the role of DA in HE, we treated HE model rats with L-DOPA. This treatment significantly increased hippocampal DA content in the model group. Interestingly, the behavioral disorders in HE rats were significantly improved, with scores and times on the beam balance test returning to normal levels among rats that received treatment. However, L-DOPA treatment had limited effects on liver recuperation. Taken together, these results further demonstrate that the decrease of hippocampal DA is related to behavioral disorders in the HE model.

Since DA improved behavioral disorders in the model group, we then explored the mechanism of this effect. The results demonstrated that D1 and D2 were both increased in hippocampus of the model group. After treatment with L-DOPA, the expression of D1 in hippocampus was reduced to normal levels, which indicates a possible role of D1 in the treatment effects. To further explore the role of D1 in the role of hippocampal $\mathrm{DA}$ in HE, we pretreated model rats with the D1 antagonist SCH23390 before L-DOPA treatment. The results showed that the D1 antagonist significantly blocked the therapeutic effects of L-DOPA in HE model rats. These results demonstrate that improvements in behavioral disorders in HE due to L-DOPA treatment are mediated by D1.

In conclusion, the present study has demonstrated that hippocampal DA plays a critical role in the pathogenesis of $\mathrm{HE}$ and that injection of L-DOPA can improve behavioral disorders in HE by activating D1 in hippocampus. Our study suggests a critical role of DA in the onset of $\mathrm{HE}$ and highlights the possible role of the DA receptor in the regulation of $\mathrm{HE}$.

\section{Conflict of Interest}

There is no conflict of interest.

\section{Acknowledgements}

This study was funded by the Natural Science Foundation of Anhui Province (1708085QH206) and Provincial Training Program of Innovation and Entrepreneurship for Undergraduates of Anhui Province (201810368067).

\section{Abbreviations}

HE - hepatic encephalopathy; DA - dopamine; L-DOPA - levodopa; TNF- $\alpha$ - tumor necrosis factor- $\alpha$; IL-10 interleukin-10; D1 - dopamine receptor 1; D2 dopamine receptor 2; WB - Western-blot; CASP-3 caspase-3; Hematoxylin-eosin staining - H\&E staining.

\section{References}

AZHARI H, SWAIN MG: Role of peripheral inflammation in hepatic encephalopathy. J Clin Exp Hepatol 8: 281-285, 2018. https://doi.org/10.1016/j.jceh.2018.06.008

ANAND KS, DHIKAV V: Hippocampus in health and disease: An overview. Ann Indian Acad Neurol 15: 239-246, 2012. https://doi.org/10.4103/0972-2327.104323

BEAUVAIS G, JAYANTHI S, MCCOY MT, LADENHEIM B, CADET JL: Differential effects of methamphetamine and $\mathrm{SCH} 23390$ on the expression of members of IEG families of transcription factors in the rat striatum. Brain Res 1318: 1-10, 2010. https://doi.org/10.1016/j.brainres.2009.12.083

BERKE JD: What does dopamine mean? Nat Neurosci 21: 787-793, 2018. https://doi.org/10.1038/s41593-018-0152-y

CAVUS I, KASOFF WS, CASSADAY MP, JACOB R, GUEORGUIEVA R, SHERWIN RS, KRYSTAL JH, SPENCER DD, ABI-SAAB WM: Extracellular metabolites in the cortex and hippocampus of epileptic patients. Ann Neurol 57: 226-235, 2005. https://doi.org/10.1002/ana.20380

CANALES JJ: Deficient plasticity in the hippocampus and the spiral of addiction: focus on adult neurogenesis. Curr Top Behav Neurosci 15: 293-312, 2013. https://doi.org/10.1007/7854_2012_230

CORDELLA A, KRASHIA P, NOBILI A, PIGNATARO A, LA BARBERA L, VISCOMI MT, VALZANIA A, KELLER F, AMMASSARI-TEULE M, MERCURI NB, BERRETTA N, D'AMELIO M: Dopamine loss alters the hippocampus-nucleus accumbens synaptic transmission in the Tg2576 mouse model of Alzheimer's disease. Neurobiol Dis 116: 142-154, 2018. https://doi.org/10.1016/j.nbd.2018.05.006 
DAVIS BC, BAJAJ JS: Effects of alcohol on the brain in cirrhosis: Beyond hepatic encephalopathy. Alcohol Clin Exp Res 42: 660-667, 2018. https://doi.org/10.1111/acer.13605

DELIS F, MITSACOS A, GIOMPRES P: Lesion of the cerebellar paravermis increases dopamine D1 receptor levels in the contralateral striatum. J Chem Neuroanat 47: 35-41, 2013. https://doi.org/10.1016/j.jchemneu.2012.10.004

DE VOOGD LD, MURRAY YPJ, BARTE RM, VAN DER HEIDE A, FERNÁNDEZ G, DOELLER CF, HERMANS EJ: The role of hippocampal spatial representations in contextualization and generalization of fear. Neuroimage: 116308, 2019. https://doi.org/10.1101/514505

DHAREL N, BAJAJ JS: Definition and nomenclature of hepatic encephalopathy. J Clin Exp Hepatol 5 (Suppl 1): S37-S41, 2015. https://doi.org/10.1016/j.jceh.2014.10.001

ELLUL, MARK A, GHOLKAR SA, CROSS TJ: Hepatic encephalopathy due to liver cirrhosis. BMJ 351: h4187, 2015. https://doi.org/10.1136/bmj.h4187

EL-GHUNDI M, O'DOWD BF, GEORGE SR: Insights into the role of dopamine receptor systems in learning and memory. Rev Neurosci 18: 37-66, 2007. https://doi.org/10.1515/REVNEURO.2007.18.1.37

GARCÍA-GARCÍA R, CRUZ-GÓMEZ ÁJ, URIOS A, MANGAS-LOSADA A, FORN C, ESCUDERO-GARCÍA D, KOSENKO E, TORREGROSA I, TOSCA J, GINER-DURÁN R, SERRA MA, AVILA C, BELLOCH V, FELIPO V, MONTOLIU C: Learning and memory impairments in patients with minimal hepatic encephalopathy are associated with structural and functional connectivity alterations in hippocampus. Sci Rep 8: 9664, 2018. https://doi.org/10.1038/s41598-018-27978-X

HADDAD F, SAWALHA M, KHAWAJA Y, NAJJAR A, KARAMAN R: Dopamine and levodopa prodrugs for the treatment of Parkinson's disease. Molecules 23: 40, 2017. https://doi.org/10.3390/molecules23010040

NINAN J, FELDMAN L: Ammonia levels and hepatic encephalopathy in patients with known chronic liver disease. J Hosp Med 12: 659-661, 2017. https://doi.org/10.12788/jhm.2794

KNELL AJ, DAVIDSON AR, WILLIAMS R, KANTAMANENI BD, CURZON G: Dopamine and serotonin metabolism in hepatic encephalopathy. Br Med J 1: 549-551, 1974. https://doi.org/10.1136/bmj.1.5907.549

KORNERUP LS, GLUUD LL, VILSTRUP H, DAM G: Update on the therapeutic management of hepatic encephalopathy. Curr Gastroenterol Rep 20: 21, 2018. https://doi.org/10.1007/s11894-018-0627-8

LI D, SONG X, HUANG H, YE Z: Association of Parkinson's disease-related pain with plasma interleukin-1, interleukin-6, interleukin-10, and tumour necrosis factor- $\alpha$. Neurosci Lett 683: 181-184, 2018. https://doi.org/10.1016/j.neulet.2018.07.027

LI F, MIAO ZN, XU YY, ZHENG SY, QIN MD, GU YZ, ZHANG XG: Transplantation of human amniotic mesenchymal stem cells in the treatment of focal cerebral ischemia. Mol Med Rep 6: 625-630, 2012. https://doi.org/10.3892/mmr.2012.968

LEKE R, BAK LK, IVERSEN P, SØRENSEN M, KEIDING S, VILSTRUP H, OTT P, PORTELA LV, SCHOUSBOE A, WAAGEPETERSEN HS: Synthesis of neurotransmitter GABA via the neuronal tricarboxylic acid cycle is elevated in rats with liver cirrhosis consistent with a high GABAergic tone in chronic hepatic encephalopathy. J Neurochem 117: 824-832, 2011. https://doi.org/10.1111/j.1471-4159.2011.07244.x

MATALKA KZ, ATTALLAH LJ, QINNA NA, ALHUSSAINY T: Dopamine selectively modulates lipopolysaccharideinduced TNF-alpha, IFN-gamma and IL-10 within mice tissues. Neuro Endocrinol Lett 32: 176-186, 2011.

OCHOA-SANCHEZ R, ROSE CF: Pathogenesis of hepatic encephalopathy in chronic liver disease. J Clin Exp Hepatol 8: 262-271, 2018. https://doi.org/10.1016/j.jceh.2018.08.001

RÖSSLE M, LUFT M, HERZ R, KLEIN B, LEHMANN M, GEROK W: Amino acid, ammonia and neurotransmitter concentrations in hepatic encephalopathy: serial analysis in plasma and cerebrospinal fluid during treatment with an adapted amino acid solution. Klin Wochenschr 62: 867-875, 1984. https://doi.org/10.1007/BF01712006

RILJAK V, LAŠTU゚VKA Z, MYSLIVEČEK J, BORBÉLYOVÁ V, OTÁHAL J: Early postnatal hypoxia induces behavioral deficits but not morphological damage in the hippocampus in adolescent rats. Physiol Res 69: 165-179, 2020. https://doi.org/10.33549/physiolres.934234

SHAWCROSS DL: Diagnosis and management of hepatic encephalopathy. Br J Nurs 27 (Suppl 3): S7-S13, 2018. https://doi.org/10.12968/bjon.2018.27.Sup3.S7

SIL'KIS IG: Possible mechanisms of learning, memory and attention impairment in consequence of sleep deprivation. (Article in Russian) Ross Fiziol Zh Im I M Sechenova 98: 1200-1212, 2012. 
SOUZA-BRAGA P, LORENA FB, NASCIMENTO BPP, MARCELINO CP, RAVACHE TT, RICCI E, BERNARDI MM, RIBEIRO MO: Adrenergic receptor $\beta 3$ is involved in the memory consolidation process in mice. Braz J Med Biol Res 51: e7564, 2018. https://doi.org/10.1590/1414-431x20187564

SCHWENDIMANN RN, MINAGAR A: Liver disease and neurology. Continuum (Minneap Minn) 23: $762-777,2017$. https://doi.org/10.1212/CON.0000000000000486

TANG J, DANI JA: Dopamine enables in vivo synaptic plasticity associated with the addictive drug nicotine. Neuron 63: 673-682, 2009. https://doi.org/10.1016/j.neuron.2009.07.025

TYCE GM, STOCKARD J, SHARPLESS NS: Excretion of amines and their metabolites by two patients in hepatic coma treated with L-dopa. Clin Pharmacol Ther 34: 390-398, 1983. https://doi.org/10.1038/clpt.1983.185

WANG WW, ZHANG Y, HUANG XB, YOU N, ZHENG L, LI J: Fecal microbiota transplantation prevents hepatic encephalopathy in rats with carbon tetrachloride-induced acute hepatic dysfunction. World J Gastroenterol 23: 6983-6994, 2017. https://doi.org/10.3748/wjg.v23.i38.6983

YANG Y, WANG JZ: From structure to behavior in basolateral amygdala-hippocampus circuits. Front Neural Circuits 11: 86, 2017. https://doi.org/10.3389/fncir.2017.00086

ZOU TX, SHE L, ZHAN C, GAO YQ, CHEN HJ: Altered topological properties of gray matter structural covariance networks in minimal hepatic encephalopathy. Front Neuroanat 12: 101, 2018. https://doi.org/10.3389/fnana.2018.00101 